\title{
Função de Sensibilidade ao Contraste: Indicador da Percepção Visual da Forma e da Resolução Espacial
}

\author{
Natanael Antonio dos Santos ${ }^{12}$ \\ Maria Lúcia de Bustamante Simas \\ Universidade Federal de Pernambuco
}

\begin{abstract}
Resumo
Este trabalho discute a função de sensibilidade ao contraste, FSC, em três seções. A primeira descreve alguns aspectos teóricos e experimentais da FSC relacionados à pesquisa básica e aplicada. A segunda discute alguns estímulos espaciais utilizados para caracterizar a FSC ou a resposta do sistema visual humano. A terceira apresenta algumas considerações finais sobre a FSC e estímulos espaciais elementares. Um dos objetivos deste trabalho é discutir a FSC que junto com a acuidade visual parecem formar os principais indicadores dos aspectos críticos da percepção visual da forma e da resolução espacial. A FSC geralmente estima a percepção de qualquer padrão em função de sua freqüência espacial.

Palavras-chaves: Percepção visual da forma; acuidade visual; função de sensibilidade ao contraste; resolução espacial; freqüência espacial.
\end{abstract}

Contrast Sensitivity Function: Indicator of the Visual Perception of Form and of the Spatial Resolution

\begin{abstract}
This work discusses the contrast sensitivity function (CSF) in three sections. The first describes some theoretical and experimental questions of the CSF related to basic and hard-working research. The second section discusses some elementary spatial stimuli used to characterize the CSF or the response of the human visual system. The third section shows some final considerations about CSF and spatial elementary stimuli. One of the goals of this paper is to discuss the CSF that together with visual acuity appear to form important indicators of the critical aspects of the visual perception of form and of the spatial resolution. The CSF normally is an estimate of the visibility of any pattern in function of the spatial frequency.

Keywords: Visual perception of form; visual acuity; contrast sensitivity function; spatial resolution; spatial frequency.
\end{abstract}

\section{Função de Sensibilidade ao Contraste}

A sensibilidade ao contraste é definida na literatura como a recíproca da quantidade mínima de contraste necessária para detectar uma grade de uma freqüência espacial específica (Cornsweet, 1970). Em outras palavras, a FSC é, por definição, o inverso da curva de limiar de contraste (1/FSC). Logo, a sensibilidade ao contraste está relacionada à diferença existente entre picos e depressões das ondas. Essa diferença traduz o valor de contraste requerido pelo sistema visual humano, SVH, para distinguir um estímulo de freqüência espacial daquele com um campo uniforme de luminância média. Assim, o sistema visual, SV, possui alta sensibilidade quando um padrão precisa de pouco contraste para ser detectado. O inverso, baixa sensibilidade, quando o SV precisa de alto valor de contraste para detectar o estímulo. A FSC ou a $1 /$ FSC geralmente estima a sensibilidade ao

\footnotetext{
${ }^{1}$ Endereço para correspondência: Laboratório de Percepção Visual, Departamento de Psicologia, $9^{\circ}$ andar, Centro de Filosofia e Ciências Humanas, UFPE, 50670-901, Recife, PE. Fone: (81) 32713734, Fax: (81) 32718271.E-mail: natanael@npd.ufpe.br

${ }^{2}$ Bolsa Pesquisador DCR/CNPq 300746/99-7 e Bolsa Pesquisador CNPq 523791/95-0 para os autores, respectivamente.
}

contraste de grades (ou qualquer padrão) como uma função de sua freqüência espacial.

\section{Modelo de Canais Múltiplos e a Função de Sensibilidade ao Contraste}

Desde que foi proposto por Campbell e Robson (1968), a abordagem de canais múltiplos tem sido utilizada para caracterizar o processamento visual da forma. Esta abordagem assume que o SVH responde seletivamente a freqüências espaciais, ou seja, o SVH processa simultaneamente diferentes faixas estreitas de freqüências espaciais (Blakemore \& Campbell, 1969b; Campbell \& Robson, 1968; Sachs, Nachmias \& Robson, 1971). Neste sentido, a abordagem de canais múltiplos ou análise de sistema linear defende que a percepção de uma cena visual qualquer ocorre porque o SVH executa uma análise ou decomposição desse padrão complexo em seus componentes mais elementares, uma espécie de análise de Fourier. Assim, de acordo com esse ponto de vista teórico, a FSC é o envelope de sensibilidade para a série total de canais, cada um sensível a uma faixa restrita e discreta do espectro de freqüência espacial (Graham \& Nachmias, 1971; Sachs e cols., 1971; Sekuler, 1974). Logo, 
para estudar estes mecanismos ou canais do ponto de vista psicofísico ou eletrofisiológico, é imprescindível mensurar primeiro a FSC ou a $1 / \mathrm{CSF}$.

\section{A Importância da Função de Sensibilidade ao Contraste}

A habilidade do homem e outros animais perceberem os detalhes dos objetos ou de uma cena visual é determinada basicamente pela capacidade de seus sistemas visuais em distinguir contraste, isto é, diferenças de brilhos de áreas adjacentes (Campbell \& Maffei, 1974). É claro que não se pode desprezar a importância, por exemplo, da configuração do estímulo, do tamanho da imagem visual projetada na retina, dentre outros fatores ópticos e psicofísicos (p. ex., erro refrativo, aberrações esféricas, acomodação, diâmetro da pupila, luminância), fisiológicos (p. ex., distribuição dos receptores na retina, fator de magnificação cortical) e psicológicos (p. ex., estado de atenção) que também exercem um papel marcante na percepção visual da forma ou na resolução espacial de detalhes. Neste sentido, uma característica marcante da FSC advém do fato que ela descreve o desempenho do sistema visual em níveis diferentes de contrastes. Isso a difere de medidas clássicas como acuidade visual, $\mathrm{AV}$, que geralmente descreve o desempenho do SVH em contraste muito alto (letras pretas sobre um fundo branco). A AV é a capacidade do SV de discriminar detalhes finos de objetos, ou ainda, o limite perceptual que considera como distintos dois pontos muito próximos (Del Rio, 1980).

Considerando que o processamento de contraste pelo SVH pode ocorrer em todos os níveis no dia a dia, inclusive podendo chegar a um ponto onde o objeto como um todo poderá não ser visto devido ao contraste muito alto ou muito baixo, a FSC é uma função muito eficiente para caracterizar a resposta do SVH. Consequentemente, determinar o limiar de contraste para estímulos com configurações distintas, como por exemplos estímulos radiais, angulares, grades senoidais, dentre outros é uma maneira diferente de caracterizar a resposta ou o comportamento do SVH.

Criteriosamente, a FSC fornece o limite entre o mundo a baixo contraste, que é, entretanto percebido, e um mundo a baixo contraste, que nunca se vê em determinadas condições. Em resumo, a FSC é considerada a descrição mais completa da função visual (Wilson, Levi, Maffei, Rovamo \& De Valois, 1990). Ela fornece um sumário rápido e proveitoso da resposta global do $\mathrm{SVH}$ para padrões de freqüências espaciais e caracteriza o processo pelo qual o sistema visual transforma informações das várias freqüências espaciais do estímulo de entrada (imput) em estímulo percebido (output).

\section{Função de Sensibilidade: Um Indicador de} Aspectos Críticos da Percepção Visual da Forma?

A AV e a FSC são funções relacionadas aos aspectos críticos da percepção visual da forma e de detalhes ou resolução espacial. A diferença é que a AV é um bom indicador dos fatores ópticos do olho, enquanto a FSC é um bom indicador, tantos dos fatores ópticos como dos fatores neurais da visão (Akutsu \& Legge, 1995; Artal, Ferro, Miranda \& Navarro, 1993; Bour \& Apkarian, 1996; Elliott \& Situ, 1998; Kiper, Gegenfurtner \& Kiorpes, 1995; Kiper \& Kiorpes, 1994; Polat, Sagi \& Norcia, 1997; Ross, Bron \& Clarke, 1984; Suter e colaboradores, 1994; Vleugels, van Nunen, Lafosse, Ketelaer \& Vandenbussche, 1998).

Desta maneira, a FSC é uma das principais funções na avaliação do SVH, quer seja do ponto de vista teórico quer seja do ponto de vista clínico (John, 1997; Kiper \& Kiorpes, 1994). Por exemplo, (1) a FSC pode ser utilizada para estudar o desenvolvimento do sistema visual ou da percepção visual da forma em crianças (Candy, Crowell \& Banks, 1998; Courage \& Adams, 1996; Van Sluyters, Atkinson, Held, Hoffman \& Shatz, 1990), pois muitos aspectos responsáveis pelo surgimento da percepção visual (p. ex., AV, FSC, visão binocular, dentre outros) estão todos reduzidos na infância, comparado ao do adulto (Brown, 1990; Van Sluyters e cols., 1990). (2) A FSC pode caracterizar mecanismos ou canais sintonizados para freqüências espaciais dentro do campo da pesquisa psicofísica e eletrofisiológica básica (Blakemore \& Campbell, 1969a; Blakemore \& Campbell, 1969b; Blakemore, Nachmias \& Sutton, 1970; Campbell \& Maffei, 1970; Campbell \& Robson, 1968; Graham \& Nachmias, 1971; Kelly \& Magnuski, 1975; Pantle \& Sekuler, 1968; Sachs e colaboradores, 1971; Santos, 1996, 1999; Simas \& Dodwell, 1990; Simas \& Santos, 1997, 1998). (3) A FSC também pode avaliar prejuízo na percepção visual de forma provocado por doenças degenerativas, desmielinização das vias visuais ou lesões corticais, como por exemplo, esclerose múltipla (Regan, Silver \& Murray, 1977), estrabismo (Kiper e cols., 1995; Kiper \& Kiorpes, 1994), albinismo (Wilson, Nagy, Mets \& Perrera, 1988), ambliopia (Polat e colaboradores, 1997; Thorn \& Comerford, 1983), catarata (Elliott \& Situ, 1998), acromatas (Rosness, Magnussen \& Nordby, 1994) e doenças de Alzheimer e de Parkinson (Akutsu \& Legge, 1995; Artal e cols., 1993; Bour \& Apkarian, 1996; Elliott \& Situ, 1998; Kiper e colaboradores, 1995; Kiper \& Kiorpes, 1994; Polat e cols., 1997; Ross e cols., 1984; Suter e cols., 1994; Vleugels e cols., 1998). Em 1984, Ross e cols. relataram que a FSC parece ser o método mais sensível para mensurar o prejuízo de pacientes com glaucoma. 
Um bom exemplo do papel da FSC ocorre na ambliopia, alteração da visão espacial devido à interação binocular anormal durante o período crítico do desenvolvimento (Polat e colaboradores, 1997). Neste caso, a FSC é importante para monitorar adequadamente os efeitos da terapia de oclusão de um dos olhos, pois o tratamento de pacientes com ambliopia envolve oclusão monocular do olho não amblíope, enquanto estimula o olho amblíope (Thorn \& Comerford, 1983).

Além de ser sensível a fatores ópticos e a alterações neuro-oftalmológicas, a CSF também é sensível ao habito comportamental do animal, isto é, mamíferos noturnos e diurnos apresentam faixas de sensibilidades diferentes (Langston, Casagrande \& Fox, 1986). Enfim, a forma e a dinâmica com que a CSF interage com as condições visualizadas permite fortes inferências sobre processos comportamentais e fisiológicos básicos.

$\mathrm{Na}$ prática, acreditamos que a AV e a FSC podem se complementar, pois pacientes com catarata inicial pode ter boa AV e reclamar de visão pobre, e isto acontece devido ao prejuízo visual estar relacionado a freqüências baixas e médias (Elliott \& Situ, 1998) e não a altas. Quando o prejuízo ocorre nas freqüências baixas e médias, a FSC é o melhor indicador da capacidade do sistema visual (Woodhouse \& Barlow, 1982). Por outro lado, a FSC tem um procedimento demorado e de difícil emprego clínico, ao passo que a AV tem um procedimento simples, rápido e muito eficaz principalmente quando o problema envolve prejuízos em freqüências altas. Este problema pode ser contornado com estímulos, isto é optotipos que levem em consideração a sensibilidade do SVH ao contraste.

\section{Estímulos Espaciais Elementares}

Os estímulos utilizados para determinar a FSC e a resposta característica de um filtro de freqüência espacial são modulações senoidais de luminância no espaço. Essas modulações periódicas são facilmente descritas no domínio da freqüência, por se constituírem de picos centrados nas freqüências referentes ao número de ciclos por unidade de espaço. Naturalmente, um estímulo modulado por apenas uma onda senoidal é descrito por apenas um pico no domínio da freqüência. Por outro lado, um estímulo não periódico pode ser descrito por uma série de picos de amplitudes sobre várias faixas de freqüências espaciais diferentes.

Por essas razões, modulações senoidais ou cossenoidais que descrevem um estímulo elementar podem ser descritas tanto por um sistema de coordenadas cartesianas como por um sistema de coordenadas polares. $\mathrm{Na}$ verdade, quando se troca o sistema de coordenadas Psicologia: Reflexão e Crítica, 2001, 14(3), pp. 589-597 cartesianas, como por exemplo, para coordenadas polares, o que muda é a definição matemática do estímulo. Em um sistema de coordenadas cartesianas, o estímulo espacial (p. ex., grade senoidal) é melhor definido em relação aos eixos $x$ e $y$, enquanto, em um sistema de coordenadas polares, o estímulo espacial é melhor definido em termos de $\theta$ e/ou $r$, onde $\theta$ representa a variável angular e $r$ a variável radial.

Apesar de se acreditar que uma das características fundamentais do SV é o reconhecimento de padrões mais complexos ou cenas visuais (De Valois \& De Valois, 1980), é freqüente a utilização de subunidades discretas ou estímulos elementares de freqüência espacial para tentar caracterizar a resposta do SVH. De certa forma, o uso de padrões simples se apóia na premissa da existência de canais múltiplos de freqüências espaciais e na idéia que o SV decompõe o estímulo complexo em componentes elementares (ondas senoidais e cossenoidais) um tipo de análise de Fourier. Além disso, um estímulo complexo pode conter muita informação, comparado a um estímulo simples, o que aumentaria o número de vias e áreas envolvidas no seu processamento, dificultando assim a localização e isolamento de mecanismos simples. Principalmente, com a possibilidade de mais da metade do córtex ser ativado por estímulos visuais (Goldstein, 1996).

\section{Estímulo Elementar Espacial Grade Senoidal}

A maioria dos estudos que procurou estimar a FSC e filtros espaciais de banda estreita utilizam grades senoidais verticais como padrão (Blakemore \& Campbell, 1969b; Campbell \& Robson, 1968; Ellemberg, Lewis, Liu \& Maurer, 1999; Maffei \& Fiorentini, 1973; Maffei \& Fiorentini, 1977; Peterzell \& Teller, 1996; Sachs e cols., 1971; Thibos, Still \& Bradley, 1996; Wilson e cols., 1990). A grade senoidal é definida em termos da modulação da amplitude de contraste e de sua freqüência espacial. Grade senoidal, portanto, é um estímulo elementar cuja luminância varia senoidalmente no espaço, em uma direção, em um sistema de coordenadas cartesianas (Figura 1). Neste caso, o perfil de luminância da freqüência é senoidal e as variáveis experimentais principais são freqüência espacial e o contraste.

Freqüência espacial é o número de ciclos (ou períodos) por medida de espaço, que em processamento visual da forma foi convencionalmente denominado de ciclo por grau de ângulo visual (cpg). Enquanto, o contraste é a relação entre a luminância máxima e luminância mínima, representado matematicamente da seguinte forma: $C=L_{\max }-L_{\min } / L_{\max }+L_{\min }$. Quando a modulação do contraste é senoidal ou cossenoidal, trata-se de 
um estímulo considerado elementar para uma análise de Fourier.

Grade senoidal é considerada, por definição, um estímulo elementar ideal para determinar as características das respostas visuais em experimentos neurofisiológicos e psicofísicos, pois foi um dos primeiros estímulos a ser utilizado para testar as propriedades de linearidade de neurônios individuais em estudos neurofisiológicos (Enroth-Cugel \& Robson, 1966; Campbell \& Maffei, 1970; Campbell, Cooper \& Enroth-Cugel, 1969a; Campbell, Cooper, Robson \& Sachs, 1969b; Maffei \& Fiorentini, 1973, 1977) e vias sintonizadas a freqüências espaciais ou canais múltiplos em estudos psicofísicos (Campbell \& Robson, 1068; Blakemore \& Campbell, 1969a, 1969b; Sachs e cols., 1971). De acordo com Thomas (1986), a introdução de grade senoidal como estímulo em estudos eletrofisiológicos e psicofísicos foi uma das metodologias mais ricas em termos de proporcionar o desenvolvimento conceptual em visão espacial.

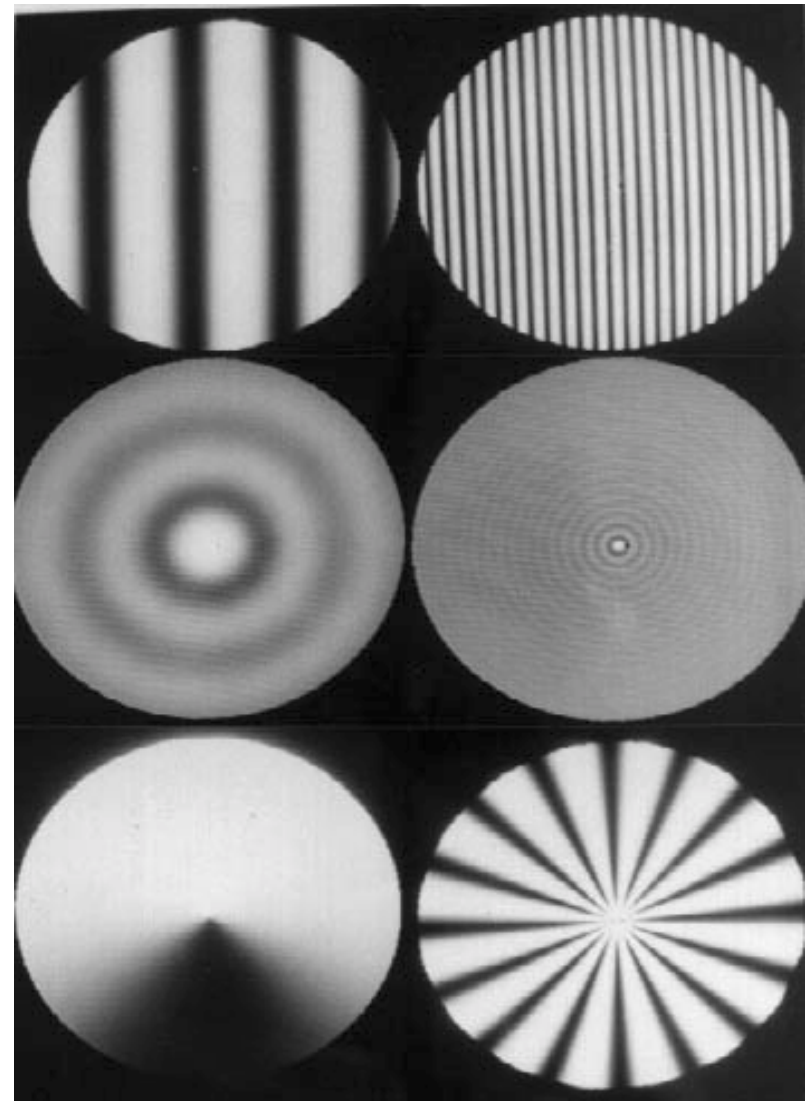

Figura 1. Exemplos de estímulos elementares de freqüências espaciais grades senoidais (acima à esquerda 0,5 cpg e à direita 3 cpg), freqüências radiais, $J_{0}$ (ao centro à esquerda 0,5 cpg e à direita $3 \mathrm{cpg}$ ) e freqüências angulares (embaixo à direita 1 ciclo e à esquerda 16 ciclos). Estímulos originalmente calibrados para serem visto a $150 \mathrm{~cm}$ de distância antes de fotografados.

\section{Estímulo Elementar de Freqüência Espacial Radial}

Em 1960, Kelly propôs o uso de estímulos circularmente simétricos, o qual ele chamou de alvos, $J_{0}$, isto é, estímulos modulado por funções cilíndricas de Bessel da ordem zero. Este padrão é denominado por nós de estímulo radial porque a modulação do contraste é dada pelo perfil da função cilíndrica de Bessel ao longo do raio considerando o centro do círculo como a origem do sistema de coordenadas polares (Figura 1).

Existem duas razões importantes acerca de padrões modulados pela função cilíndrica de Bessel; primeira, este padrão simetricamente circular em geral parece ser mais natural considerando a forma aproximadamente circular e simétrica da retina; e segunda, o fato particular de $\mathrm{J}_{0}$ estabelecer um centro de fixação claro o que outros padrões, como por exemplo a grade senoidal, não fornecem (Kelly, 1960; Kelly \& Magnuski, 1975).

Diferente da grade senoidal que tem sua oscilação de contraste entre máximo e mínimo no mesmo intervalo fixo, o estímulo circular proposto por Kelly (1960) tem contraste máximo no centro, decrescendo gradualmente na direção radial (periferia), ou seja, a amplitude de cada círculo em uma dada freqüência diminui à medida que se afasta do centro para a periferia do campo visual (Figura 1). Assim como, em um raio fixado, a amplitude local do círculo diminui quando a sua freqüência aumenta (Kelly \& Magnuski, 1975). Estas características podem ser importantes para caracterizar a resposta do SV de animais diurnos que possuem fóvea bem definida.

\section{Estímulo Elementar de Freqüência Espacial Angular}

Em 1985, Simas introduziu a idéia de estímulos elementares de freqüências angulares como o componente ortogonal a freqüências radiais (Eq\# 1-5). Pois apesar de adotar coordenadas polares, Kelly (1960) em sua proposta inicial sugeriu apenas a coordenada radial $(r)$ ignorando a direção ortogonal, isto é, a coordenada angular $(\theta)$.

O estímulo freqüência angular é um padrão cuja luminância varia, de acordo com o seno ou o cosseno, na direção angular em um sistema de coordenadas polares. Neste caso, a fase da freqüência angular varia entre 0 (zero) e $2 \mathrm{p}$ e a freqüência é discreta e sempre dada por número inteiro de ciclos por $360^{\circ}$ (Figuras 1 e 2). Assim, o estímulo angular é adimensional, inteiro e sua freqüência independe da distância.

Estímulos de freqüência angular aparecem na literatura com uma variedade de nomes, por exemplo, alvos radiais ou radial targets, grades polares ou polar gratings, estímulos moinho de vento ou windmill stimuli, forma de 
estrela ou star-like, dentre outros. Por consistência, como a freqüência angular varia senoidalmente (ou cossenoidalmente, dependendo da fase) com o ângulo em um círculo a nomenclatura de estímulo de freqüência angular por nós utilizada parece ser mais apropriada.

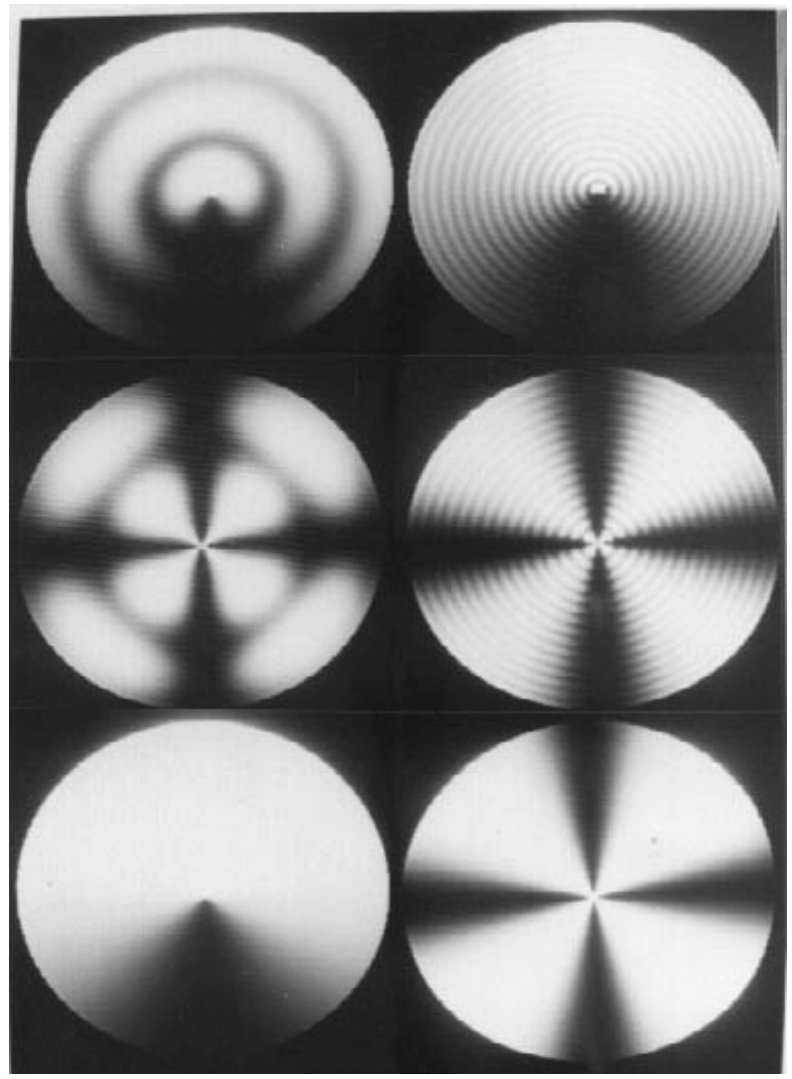

Figura 2. Exemplos de estímulos de freqüências radiais e angulares acoplados. Acima à esquerda e à direita, as freqüências radiais de 0,5 cpg e 3 cpg acopladas à freqüência angular de 1 ciclo, respectivamente. No centro à direita e à esquerda as freqüências radiais 0,5 cpg e 3 cpg acopladas à freqüência angular de 4 ciclos. Embaixo, as freqüências angulares de 1 e 4 ciclos, respectivamente. Estímulos originalmente calibrados para serem vistos a $150 \mathrm{~cm}$ de distância antes de fotografados.

\section{Estímulo Espacial de Freqüência Radial/Angular Acoplada}

A introdução de estímulo de freqüência radial e angular acoplado em coordenadas polares (Santos, 1996, 1999; Simas \& Santos, 1997, 1998) foi possível graças a retificação da equação de Sneddon, Eq \# 1 (Sneddon, 1961) que permite acoplar componentes radiais a angulares gerando padrões de forma simétrica $(E q \# 5$ e Figura 2).

As equações 1-5 descrevem uma série de Hankel em coordenadas polares e demonstram uma maneira onde ambos os componentes, radial e angular, podem ser descritos como estímulos elementares em um sistema de coordenadas polares com freqüência angular e/ou radial simples ou acopladas pelo mesmo valor da ordem (n) da função de Bessel. Neste caso, o núcleo da série de Fourier (para estímulos elementares acoplados através dos dois componentes) assume a forma funcional de Sneddon (1961):

$$
\exp (i x \operatorname{sen} \theta)=\sum_{n=-\infty}^{\infty} J_{n}(x) e^{i n} \theta \quad \text { [Eq.\# 1] }
$$

onde $J_{n}(x)$ é a parte radial e $e^{i n} \boldsymbol{\theta}$ a parte angular, substituindo $x$ por $2 \pi \rho r$, tem-se:

$$
\exp (i 2 \pi \rho r \operatorname{sen} \theta)=\sum_{n=-\infty}^{\infty} J_{n}(2 \pi \rho r) e^{i n} \theta \quad[E q . \# 2]
$$

onde:

$$
e^{i n} \theta=\cos (n \theta)+i \operatorname{sen}(n \theta) \quad[E q . \# 3]
$$

assim:

$$
\exp (i 2 \pi \rho r \operatorname{sen} \theta)=\sum J_{n}(2 \pi \rho r)\{\cos (n \theta)+i \operatorname{sen}(n \theta)\}[E q . \# 4]
$$

Retificando a Eq\# 4, ou seja, substituindo a parte real do componente ortogonal (angular) $\cos (n \theta)$ pelo seu módulo, $|\cos (n \theta)|$, tem-se:

$$
\exp (i 2 \pi p r s e n \theta)=\sum J_{n}(2 \pi p r)\left\{\begin{array}{c}
\infty \\
n=-\infty \\
n=-\infty
\end{array}\right.
$$

que é denominado de retificação de onda completa. A retificação da equação é importante porque, assim, origina-se padrões espaciais simétricos de freqüências radiais e angulares acopladas. Estes padrões de simetria par podem ser importantes para estudar o processamento e reconhecimento de objetos simétricos complexos, como por exemplo faces, e para medir a sensibilidade de células em áreas do córtex visual sensíveis a estímulos simétricos (ver consideração final).

Os estímulos radiais, radiais/angulares acoplados e grade senoidais são diferentes do estímulo angular, pois as suas freqüências podem ser inteiras ou não, são dimensionais e definidos como ciclo por grau de ângulo visual, portanto caracterizando uma freqüência espacial que depende da distância do voluntário.

\section{Outros Estímulos de Freqüências Espaciais}

A literatura demonstra que existe uma variedade de outras funções na matemática que podem ser ajustadas para estudar freqüência espacial e caracterizar a resposta do SV. Como exemplo, pode-se mencionar a função de 
Gabor, uma vez que é muito utilizada para estudar a análise de freqüência espacial local (Daugman, 1984; Geri, Lyon \& Zeevi, 1995; Graham, Sutter \& Venkatesan, 1993; Hess \& Hayes, 1994; Hess \& Wilcox, 1994; Polat \& Sagi, 1993; Pollen, Nagler, Daugman, Kroaner \& Koenderink, 1984; Sutter, Sperling \& Chubb, 1995).

A função de Gabor $G(x, y)$ para um filtro vertical é definida como:

$$
G(x, y)=A \operatorname{sen}(2 \pi \omega x+\phi) e^{-x^{2} / s_{x}^{2}-y^{2} /{ }_{s}^{2} .}
$$

De acordo com Wilson e colaboradores (1990), essa é apenas uma onda senoidal de fase variável multiplicada por uma função Gaussiana bidimensional que pode ter diferentes constantes nas direções $x$ e $y$. Dependendo da fase $\mathrm{f}$ da função de Gabor, a mesma pode ter simetria ímpar ou par, ou pode ser assimétrica.

Existem outros trabalhos na literatura utilizando sistemas de coordenadas polares para descrever o processo operacional realizado pelo sistema visual. Estes incluem outros tipos de padrões visuais, por exemplo: grades, padrões xadrez, "olho de touro", radial, angular, textura, padrões em coordenadas polares e cartesianas formados por pontos aleatórios, estímulos do tipo "glass", dentre outros (Carlson, Cohen \& Gorog, 1977; Cavanagh, 1978; Cavanagh, 1982; Dodwell, 1983; Gallant, Brau \& van Essen, 1993; Gallant, Connor, Rakshit, Lewis \& van Essen, 1996; Hess, Wang, Demanins, Wilkinson \& Wilson, 1999; Hess \& Wilcox, 1994; Rosa, Gattass, Fiorani Jr \& Soares, 1992; Schwartz, 1983; Verrall \& Kakarala, 1998; Weisstein, Harris, Berbaum, Tangney \& Williams, 1977; Wilkinson, Wilson \& Habak, 1998; Wilson \& Wilkinson, 1997, 1998).

\section{Considerações Finais sobre a CSF e Estímulos Espaciais Elementares}

A aplicação da análise de sistemas lineares ou análise de Fourier à visão tem sido extensamente utilizada desde os estudos pioneiros de Schade (1948) e Selwyn (1948). Entretanto, a análise de Fourier tem se restringido, na maioria absoluta dos casos, ao emprego de estímulos elementares em coordenadas cartesianas para caracterizar a resposta do sistema visual. Neste contexto, foram poucas as propostas que tentaram caracterizar o SVH como processando a informação espacial em termos que poderiam ser melhor descritos em um sistema de coordenadas polares pegando áreas inteiras do campo visual. Apesar de nos últimos três anos, o número de trabalhos que usam estímulos em coordenadas polares, haver aumentado significativamente (Amidror, 1997; Hess e cols., 1999; Verrall \& Kakarala, 1998; Wilkinson e cols., 1998; Wilson \& Wilkinson, 1997, 1998; Wilson, Wilkinson
\& Asaad, 1997). Estes trabalhos têm perspectivas teóricas diferentes de Kelly (1960) e Simas (1985).

Alguns trabalhos com sugestões teóricas a favor da abordagem de canais múltiplos e filtragem de áreas inteiras do campo visual merecem destaque: (1) Kelly e Magnuski (1975) que mensuraram pela primeira vez a FSC para estímulo radiais; (2) Simas e Dodwell (1990) e Simas, Santos e Thiers (1997) que mensuraram a FSC para estímulos angulares; e (3) Santos (1996, 1999) e Simas e Santos $(1997,1998)$, que não só retomaram os estudos de Kelly mensurando a FSC para estímulos radiais modulados pela função cilíndrica de Bessel, mas também mensuram pela primeira vez a FSC para estímulos radiais modulados pela função esférica de Bessel. Estes autores mediram também a FSC para estímulos radiais/angulares acoplados.

Podemos também chamar a atenção para os estudos com macaco de Gallant e colaboradores (1993, 1996) que introduziram na literatura estímulos com configurações espaciais diferentes e encontraram evidências eletrofisiológicas para detecção de estímulos em coordenadas polares em áreas visuais extra-estriado. Por exemplo, Gallant e colaboradores (1993) mediram as respostas de células na área V4 de macaco para três classes de estímulos periódicos de modulação quadrada: concêntrico e angular (polar), hiperbólicos e grades senoidais (cartesiano). Os resultados mostram que das 118 células testadas, $16 \%$ responderam significativamente mais para estímulos polares e hipérboles (grades não cartesianas) do que grades cartesianas, e apenas $8 \%$ mostraram uma percentagem significativa para grades cartesianas. Entre as células seletivas para grades não cartesianas, aquelas que preferiram grades concêntricas foram mais comuns.

No contexto atual, existe a preocupação de encontrar aspectos teóricos que possam relacionar a FSC para estímulos em coordenadas cartesianas e coordenadas polares ao processamento espacial de áreas ou faixas grandes do campo visual (Wilkinson e cols., 1998; Wilson \& Wilkinson, 1997, 1998; Wilson e cols., 1997) e o processamento visual de faces (Santos, 1999; Simas \& Santos, 1997, 1998). Acredita-se que estímulos radiais e angulares circularmente simétricos acoplados ou não podem render frutos que outros estímulos, como por exemplos grade senoidal, função de Gabor, dentre outros, não rendem (Santos, 1999). É possível que estímulos com configurações espaciais distintas possam ter mecanismos distintos envolvidos no seu processamento espacial. Neste sentido, existem fortes evidências, baseadas em estudos psicofísicos e neurofisiológicos, de que o processamento 
linear ou filtragem espacial local sintonizado para orientação e freqüência espacial de estímulos projetados na retina ocorra nos estágios iniciais da visão, isto é, em neurônios do córtex visual primário, V1 (De Valois \& De Valois, 1988; Wilson \& Wilkinson, 1998; Wilson e cols., 1997). Enquanto, por outro lado, existem dados baseados em estudos psicofísicos (Wilkinson e cols., 1998; Wilson \& Wilkinson, 1997, 1998; Wilson e cols., 1997) e neurofisiológicos (Bruce, Desimone \& Gross, 1981; Desimone, 1991; Desimone \& Schein, 1987; Gallant e colaboradores, 1993; 1996; Heywood, Gadotti \& Cowey, 1992; Merigan, 1996; Van Essen, Anderson \& Felleman, 1992; Young, 1992), de que o processamento da informação que ocorre em vias intermediárias (p. ex. área V4) e áreas mais avançadas (p. ex. córtex IT) do sistema visual são modeladas por filtros espaciais globais em coordenadas polares. Alguns desses trabalhos citados acima destacam que a área V4 pode formar o principal estágio intermediário da visão da forma de V1 para IT (Heywood e cols., 1992; Merigan, 1996; Van Essen e cols., 1992; Young, 1992). Assim, é possível que a filtragem e integração de padrões em coordenadas polares ocorram em áreas visuais extra-estriado (Ex.: V4 e IT).

Os tópicos aqui discutidos formam parte da base teórica de nossa pesquisa sobre percepção e processamento visual da forma na qual temos procurado fundamentar nossos trabalhos com humanos e métodos psicofísicos.

\section{Referências}

Akutsu, H. \& Legge, G. E. (1995). Discrimination of compound gratings: Spatial-frequency channels or local features? Vision Research, 35, 26852695.

Amidor, I. (1997). Fourier spectrum of radially periodic images. Journal of the Optical Society of America A, 14, 816-826.

Artal, P., Ferro, M., Miranda, I. \& Navarro, R. (1993). Effects of aging in retinal image quality. Journal of the Optical Society of America A, 10, 16561662.

Blakemore, C. \& Campbell, F. C. (1969a). Adaptation to spatial stimuli. Journal of Physiology, 200, 11-12p.

Blakemore, C. \& Campbell, F. C. (1969b). On the existence of neurons in the human visual system selectively sensitive to the orientation and size of retinal images. Journal of Physiology, 203, 237-260.

Blakemore, C., Nachmias, J. \& Sutton, P. (1970). The perceived spatial frequency selective neurones in the human brain. Journal of Physiology, $210,727-750$.

Bour, L. J. \& Apkarian, P. (1996). Selective broad-band spatial frequency loss in contrast sensitivity functions. Investigative Ophthalmology \& $\mathrm{Vi}$ sual Science, 37(12), 2475-2484.

Brown, A. M. (1990). Development of visual sensitivity to light and color vision in infants: A critical review. Vision Research, 30, 1159-1188.

Bruce, C. J., Desimone, R. \& Gross, C. G. (1981). Visual properties of neurons in a polysensory area in superior temporal sulcus of the macaque. Journal of Neurophysiology, 46, 369-384.
Campbell, F. W., Cooper, G. F. \& Enroth-Cugell, C. (1969a). The spatial selectivity of the visual cells of the cat. Journal of Physiology, 203, 223235.

Campbell, F. W., Cooper, G. F., Robson, J. G. \& Sachs, M. B. (1969b). The spatial selectivity of cells of the cat and the squirrel monkey. Journal of Physiology, 204, 120-121P.

Campbell, F. W. \& Maffei, L. (1970). Electrophysiological evidence for the existence of orientation and size detectors in the human visual system. Journal of Physiology, 207, 635-652.

Campbell, F. W. \& Maffei, L. (1974). Contrast and spatial frequency. Scientific American, 231, 106-114.

Campbell, F. W. \& Robson, F. G. (1968). Application of the Fourier analysis to the visibility of gratings. Journal of Physiology, 197, 551-566.

Candy, T. R., Crowell, J. A. \& Banks, M. S. (1998). Optical, receptoral, and retinal constraints on foveal and peripheral vision in the human neonate. Vision Research, 38, 3857-3870.

Carlson, C. R., Cohen, R. W. \& Gorog, I. (1977). Visual processing of simple two-dimensional sine-wave gratings. Vision Research, 17, 351-358.

Cavanagh, P. (1978). Size and position invariance in the visual system. Perception, 7, 167-177.

Cavanagh, P. (1982). Functional size invariance is not provided by cortical magnification factor. Vision Research, 22, 1409-1412.

Cornsweet, T. N. (1970). Vision perception. New York: Academic Press.

Courage, M. L. \& Adams, R. J. (1996). Infant peripheral vision: The development of monocular visual acuity in the first 3 months of postnatal life. Vision Research, 36, 1207-1215.

Daugman, J. G. (1984). Spatial visual channels in the Fourier plane. Vision Research, 24(9), 891-910.

Del Rio, E. G. (1980). Optica fisiologica clinica. Barcelona: Toray S.A.: Fourth Edition.

De Valois, R. L. \& De Valois, K. K. (1980). Spatial vision. Annual Review of Psychology, 31, 309-341.

De Valois, R. L. \& De Valois, K. K. (1988). Spatial vision. New York: Oxford University Press.

Desimone, R. (1991). Face-selective cells in the temporal cortex of monkeys. Journal of Cognitive Neuroscience, 3, 1-8.

Desimone, R. \& Schein, S. J. (1987). Visual properties of neurons in area V4 of macaque: Sensitivity to stimulus form. Journal of Neurophysiology, 57, 835-867.

Dodwell, P. C. (1983). The lie transformation group model of visual perception. Perception \& Psychophysics, 34, 1-16.

Ellemberg, D., Lewis, T. L., Liu, C. H. \& Maurer, D. (1999). Development of spatial and temporal vision during childhood. Vision Research, 39, 2325-2333

Elliott, D. B. \& Situ, P. (1998). Visual acuity versus letter contrast sensitivity in early cataract. Vision Research, 38, 2047-2052.

Enroth-Cugell, C. \& Robson, J. M. (1966). The contrast sensitivity of retinal ganglion cells of the cat. Journal of Physiology, 187, 512-552.

Gallant, J. L., Brau, J. \& van Essen, D. C. (1993). Selectivity for polar hyperbolic, and cartesian gratings in macaque visual cortex. Science, 259, 100-103.

Gallant, J. L., Connor, C. E., Rakshit, S., Lewis, J. W. \& van Essen, D. C. (1996). Neural responses to polar, hyperbolic, and cartesian gratings in area V4 of the macaque monkey. Journal of Neurophysiology, 76, 27182739.

Geri, G. A., Lyon, D. R. \& Zeevi, Y. Y. (1995). Preattentive equivalence of multicomponent Gabor textures in the central and peripheral visual field. Vision Research, 35, 495-506.

Goldstein, E. B. (1996). Sensation \& perception. New York: Brooks/Cole.

Graham, N. \& Nachmias, J. (1971). Detection of grating patterns containing two spatial frequencies: A comparison of single-channel and multiple channel models. Vision Research, 11, 251-259.

Graham, N., Sutter, A. \& Venkatesan, C. (1993). Spatial-frequency - and orientation-selectivity of simple and complex channels in region segregation. Vision Research, 33, 1893-1911. 
Hess, R. F. \& Hayes, A. (1994). The coding of spatial position by human visual system: Effects of spatial scale and retinal eccentricity. Vision Research, 34, 625-643.

Hess, R. F., Wang, Y.-Z., Demanins, R., Wilkinson, F. \& Wilson, H. R. (1999). A deficit in strabismic amblyopia for glabal shape detection. Vision Research, 39, 901-914.

Hess, R. F. \& Wilcox, L. M. (1994). Linear and nor-linear filtering stereopsis. Vision Research, 34, 2431-2438.

Heywood, C. A., Gadotti, A. \& Cowey, A. (1992). Cortical area V4 and its role in the perception of color. Journal of Neuroscience, 12, 4056-4065.

John, R. S. (1997). Contrast detection and orientation discrimination thresholds associated with meridional amblyopia. Vision Research, 37, 1451-1457.

Kelly, D. H. (1960). Jo stimulus patterns for vision research. Journal of the Optical Society of America, 50, 1115-1116.

Kelly, D. H. \& Magnuski, H. S. (1975). Pattern detection and the two demensional Fourier transform: Circular targets. Vision Research, 15, 911-915.

Kiper, D. C., Gegenfurtner, K. R. \& Kiorpes. (1995). Spatial frequency channels in experimentally strabismic monkeys revealed by oblique masking. Vision Research, 35, 2737-2742.

Kiper, D. C. \& Kiorpes. (1994). Suprathreshold contrast sensitivity in experimentally strabismic monkeys. Vision Research, 34, 1575-1583.

Langston, A., Casagrande, V. A. \& Fox, R. (1986). Spatial resolution of the galago. Vision Research, 26, 791-796.

Maffei, L. \& Fiorentini, A. (1973). The visual cortex as a spatial frequency analyzer. Vision Research, 13, 1255-1267.

Maffei, L. \& Fiorentini, A. (1977). Spatial frequency rows in the striate visual cortex. Vision Research, 17, 257-264.

Merigan, W. H. (1996). Basic visual capabilities and shape discrimination after lesions of extrastriate area V4 in macaques. Visual Neuroscience, 13, 51-60.

Pantle, A. \& Sekuler, R. (1968). Size detecting mechanisms in human vision. Science, 162, 1146-1148.

Peterzell, D. H. \& Teller, D. Y. (1996). Individual differences in contrast sensitivity functions: The lowest spatial frequency channels. Vision Research, 36, 3077-3085.

Polat, U. \& Sagi, D. (1993). Lateral interactions between spatial channels: Suppression and facilitation revealed by lateral masking experiments. Vision Research, 33, 993-999.

Polat, U., Sagi, D. \& Norcia, A. M. (1997). Abnormal long-range spatial interactions in amblyopia. Vision Research, 37, 737-744.

Pollen, D. A., Nagler, M., Daugman, J. G., Kroaner, R. \& Koenderink, J. J. (1984). Use of Gabor elementary functions to probe receptive field substructure of posterior inferotemporal neurons in the owl monkey. Vision Research, 24, 233-242.

Regan, D., Silver, R. \& Murray, T. J. (1977). Visual acuity and contrast sensitivity in multiple sclerosis-hidden visual class. Brain, 100, 563-579.

Rosa, M. G. P., Gattass, R., Fiorani Jr, M. \& Soares, J. G. M. (1992). Laminar, columnar and topographic aspects of ocular dominance in the primary visual cortex of Cebus monkeys. Experimental Brain Research, 88, 249264.

Rosness, R., Magnussen, S. \& Nordby, K. (1994). Spatial vision of the achromatic: The tilt aftereffect. Vision Research, 34, 2021-2022.

Ross, J. E., Bron, A. J. \& Clarke, D. D. (1984). Contrast sensitivity and visual disability in chronic simple glaucoma. British Journal of Ophthalmology, 68(11), 821-827.

Sachs, M. B., Nachmias, J. \& Robson, J. G. (1971). Spatial frequency channels in human vision. Journal of the Optical Society of America, 61, 1176-1186.

Santos, N. A. (1996). Sistema visual bumano: Filtragem de freqüências radiais moduladas por perfis de Bessel j0, j1, j2, j4, j8 e j16. Dissertação de Mestrado não-publicada, Curso de Pós-Graduação em Neurociências e Comportamento, Instituto de Psicologia, Universidade de São Paulo. São Paulo, Brasil.
Santos, N. A. (1999). Sistema visual humano: Curvas de sensibilidade e filtragem de freqüencias angulares, radiais e radial/ angulares acopladas. Tese de Doutorado não-publicada, Curso de Pós-Graduação em Neurociências e Comportamento, Instituto de Psicologia, Universidade de São Paulo. São Paulo, Brasil.

Schade, O. H. (1948). Electro-optical characteristics of television systems. 1. Characteristics of vision and visual systems. RCA Reviem, 9, 5-37.

Schwartz, E. L. (1983). Cortical mapping and perceptual invariance: A reply to Cavanagh. Vision Research, 23, 831-835.

Sekuler, R. (1974). Spatial vision. Annual Review of Psychology, 25, 195-232.

Selwyn, E. W. H. (1948). The photographic and visual resolving power of lenses. 1. Visual resolving power. Photographic Journal, 88B, 6-12.

Simas, M. L. B. (1985). Linearity and domain invariance in the visual system. Tese de Doutorado não publicada. Queen's University at Kingston, Ontario, Canada: University Microfilms International. Ann Arbor: Michigan.

Simas, M. L. B. \& Dodwell, P. C. (1990). Angular frequency filtering: A basis for pattern decomposition. Spatial Vision, 5, 59-74.

Simas, M. L. B. \& Santos, N. A. (1997). Human visual contrast detection of radial frequency stimuli defined by Bessel profiles $j 0, j 1, j 2, j 4, j 8$ and j16 and its relation to angular frequency. Proceedings of the II Workshop on Cybernetic Vision, IEEE Computer Science, 219-224.

Simas, M. L. B. \& Santos, N. A. (1998). Human frequency response functions of harmonic 2, 4, 8, and 16 cycle angular frequency filters. Proceedings of the Internacional Symposium on Computer Graphics, Image Processing and Vision, IEEE Computer Science, 312-319.

Simas, M. L. S., Santos, N. A. \& Thiers, F. A. (1997). Contrast sensitivity to angular frequency stimuli is higher than that for sinewave gratings in the respective middle range. Brazilian Journal Medical Biological Reserach, 30, 633-636.

Sneddon, I. N. (1961). Special functions of mathematical physics and chemistry. New York: Interscience.

Suter, P. S., Suter, S., Roessler, J. S., Parker, K. L., Armstrong, C. A. \& Powers, J. C. (1994). Spatial-frequency-tuned channels in early infancy: VEP evidence. Vision Research, 34, 737-745.

Sutter, A., Sperling, G. \& Chubb, C. (1995). Measuring the spatial frequency selectivity of second-order texture mechanisms. Vision Research, 35, 915-924.

Thibos, L. N., Still, D. L. \& Bradley, A. (1996). Characterization of spatial aliasing and contrast sensitivity in peripheral vision. Vision Research, $36,249-258$.

Thomas, J. P. (1986). Spatial vision then and now. Vision Research, 26, 15231530.

Thorn, F. \& Comerford, J. P. (1983). Use of various measures of visual acuity and contrast sensitivity in the evaluation of monocular occlusion and active vision training of three adult amblyopes. American Juornal of Optometry and Physiological Optcs, 60, 426-435.

Van Essen, D. C., Anderson, C. H. \& Felleman, D. J. (1992). Information processing in the primate visual system: An integrated systems perspective. Science, 255, 419-423.

Van Sluyters, R. C., Atkinson, M. S., Held, R. M., Hoffman, K. \& Shatz, C. J. (1990). The development of vision and visual perception. Em S. W. Spillmann \& J. S. Werner (Orgs.), The neurophysiological foundations (pp. 349-379). New York: Academic Press.

Verrall, S. C. \& Kakarala, R. (1998). Disk-harmonic coefficients for invariant pattern recognition. Journal of the Optical Society of America A, 15, 389401.

Vleugels, L., van Nunen, A., Lafosse, C., Ketelaer, P. \& Vandenbussche, E. (1998). Temporal and spatial resolution in foveal vision of multiple sclerosis patients. Vision Research, 38, 2987-2997.

Weisstein, N., Harris, C. S., Berbaum, K., Tangney, J. \& Williams, A. (1977). Contraste reduction by small localized stimuli: Extensive spatial spread of above-threshold orientation-selective masking. Vision Research, 17, 341-350.

Wilkinson, F., Wilson, H. R. \& Habak, C. (1998). Detection and recognition of radial frequency patterns. Vision Research, 38, 3555-3568. 
Wilson, H. R., Levi, D., Maffei, L., Rovamo, J. \& De Valois, R. (1990). The perception of form: Retina to striate cortex. Em S. W. Spillmann \& J. S. Werner (Orgs.), Visual perception: The neurophysiological foundation (pp. 231-271). New York: Academic Press.

Wilson, H. R., Nagy, S. E., Mets, M. B. \& Perrera, V. P. (1988). Spatial frequency and orientation tunning of spatial visual mechanisms in human albinos. Vision Research, 28, 991-999.

Wilson, H. R. \& Wilkinson, F. (1997). Evolving concepts of spatial channels in vision: From independence to nonlinear interactions. Perception, 26, 939-960.

Wilson, H. R. \& Wilkinson, F. (1998). Detection of global structure in glass patterns: Implications for form vision. Vision Research, 38, 29332947.
Wilson, R. W., Wilkinson, F. \& Asaad, W. (1997). Concentric orientation summation in human form vision. Vision Research, 37, 2325-2330.

Woodhouse, J. M. \& Barlow, H. B. (1982). Saptial and temporal resolution and analysis. Em H.B. Barlow \& J.D. Mollon (Orgs.), The senses (pp. 133164). Cambridge University Press.

Young, M. P. (1992). Objective analysis of topological organization of the primate cortical visual system. Nature, 358, 152-155.

Sobre os autores

Natanael Antonio dos Santos é Psicólogo, Pesquisador do CNPq, Bolsa DCR, Doutor em Neurociências e Comportamento pelo Instituto de Psicologia Experimental da Universidade de São Paulo (1999), Professor pesquisador do Curso de Psicologia da Universidade Federal de Pernambuco, Recife, PE.

Maria Lúcia de Bustamante Simas é Psicólogo, Pesquisador de CNPq, Doutora em Psicologia pela Queen's University, Ontario, Canadá (1985), Professora Adjunta do Curso de Psicologia da Universidade Federal de Pernambuco, Recife, PE. 